\title{
A Radio Resource Management Framework for QoS Support in Multiservice WiMAX Networks
}

\author{
A. Molinaro and S. Pizzi \\ DIMET, University "Mediterranea" of Reggio Calabria \\ Via Graziella, Loc. Feo di Vito, 89100, Reggio Calabria, Italy \\ Email: [antonella.molinaro|sara.pizzi]@unirc.it
}

\begin{abstract}
Among enabling technologies for the fourth generation networks, WiMAX represents a valuable solution because of its ability to provide wideband coverage and differentiated treatment to traffic with heterogeneous Quality of Service $(\mathrm{QoS})$ requirements. This paper focuses on the design of a channeladaptive QoS-supporting framework for radio resource management in WiMAX networks with fixed subscriber stations. The base station generates resource grants on the basis of (i) flowbased bandwidth requests, (ii) monitored link conditions to each subscriber station, and (iii) minimum resource requirements by high-demanding flows. A compensation technique that mitigates the negative effects caused by impairments on the radio channel is also presented. The effectiveness of the proposed solution in supporting both inter-class traffic QoS differentiation and intraclass fairness is demonstrated through simulations.
\end{abstract}

Index Terms-WiMAX, Quality of Service, Scheduling, Channel awareness

\section{INTRODUCTION}

Fourth generation wireless networks are expected to support heterogeneous traffic with different Quality of Service (QoS) requirements. Among broadband wireless access technologies, WiMAX (Worldwide Interoperability for Microwave Access), specified in the IEEE 802.16 standard [1], represents a valuable solution because of its ability to provide large data volumes to a high number of users distributed over wide areas in a cost-effective manner.

The design of effective radio resource management (RRM) algorithms for WiMAX networks is mandatory and very challenging, not only because the applications to be delivered are high-bandwidth demanding and have stringent delay constraints, but also because of the wireless operating environment in which WiMAX networks operate. The space- and timevarying nature of the wireless channel can be an impediment for the correct reception of data packets. Nevertheless, the success of the WiMAX technology is strictly related to its capability of providing service differentiation among different traffic classes and intra-class fairness, while assuring minimum performance guarantees to high demanding flows in the challenging wireless environment.

Although some QoS design principles are advocated in the standard [1], several implementation details are left open. In this paper, we analyze the problem of managing the uplink resources of a WiMAX network while providing QoS differentiation and fairness among traffic flows. We propose a RRM framework where the base station (BS) grants radio resource allocation on the basis of (i) flow-based bandwidth requests received from the subscriber stations (SSs), (ii) currently perceived quality on each SS-to-BS link, and (iii) minimum resource requirements by high-demanding flows like realtime traffic. An effective technique that mitigates the negative effects caused by the wireless channel and compensates for lost transmission opportunities is also proposed, with the aim of improving intra-class fairness and providing each flow with the requested minimum performance assurance.

The paper is organized as follows. The next section presents a brief overview of the research work in the area of scheduling for WiMAX networks. In section III the technical fundamentals of WiMAX are summarised. The proposed RRM framework is described in section IV and performance results are analyzed in section $\mathrm{V}$. Concluding remarks are given in the last section.

\section{RELATED WORK}

The topic of scheduling in WiMAX networks has been widely debated in the literature. Works in this field can be classified on the basis of: (i) the link direction in which they operate (uplink or downlink), (ii) the channel-aware or unaware nature of the proposal, (iii) the homogeneous or hybrid structure of the scheduling architecture, and (iv) the utilized approach to the scheduling problem.

Regarding the first type of classification, the problems of scheduling downlink data queues and uplink bandwidth requests are substantially different. In the first case, the BS can gather per-packet information, while only information on per-flow bandwidth requirements are available to the BS when scheduling resource requests from the SSs. With regards to the scheduling of uplink traffic, in [2] a predictive algorithm is proposed to be applied on the queues of bandwidth requests in the BS; it aims at minimizing the delay for the real-time polling service (rtPS) flows based on the calculation of a per-flow deadline. In both [3] and [4] the uplink scheduling problem is examined as two separate sub-problems: inter-class and intra-class scheduling. In [3] the authors propose a solution for a combined scheduling of uplink and downlink using the concept of virtual packets. In [4] inter-class scheduling is carried out through a RED-Based Weighted Fair Priority Queuing, while a long-term proportional fair algorithm based on Maximum Signal to Interference Ratio is used for intraclass scheduling. 
Since channel-awareness is mandatory in order to carry effective resource allocation [5], many research works have developed scheduling algorithms that keep into account the channel state information [6]. A recent survey of research in scheduling for WiMAX networks, classified on the basis of the usage of channel status information, is presented in [7].

In [8] scheduling algorithms in WiMAX are classified in three categories: homogenous, hybrid and opportunistic. While homogeneous algorithms have been proposed in [9], in [10] the authors define a hybrid uplink scheduling algorithm for the SSs that is the combination of priority-based and Earliest Due Date scheduling methods. Instead, the Earliest Deadline First scheduling algorithm is proposed in [11] for the management of uplink rtPS flows to avoid the delay violation.

Also different approaches are applied to resource allocation, such as game theory [12] and optimization techniques [13].

Compared with these approaches, our proposal for radio resource management in WiMAX: (i) considers channelawareness as a mandatory feature, (ii) provides minimum performance guarantees to high demanding traffic flows, (iii) works without requiring further information from the SSs besides the bandwidth requests, and (iv) considers all the traffic classes foreseen by the standard.

\section{WiMAX TECHNOLOGY}

WiMAX networks are centrally managed by the BS that offers connection-oriented services to fixed SSs. Both Time Division Duplexing (TDD) and Frequency Division Duplexing (FDD) modes can be used. In this paper, we refer to TDD, but the principles of the proposal are valid regardless of the duplexing method.

At the Medium Access Control (MAC) layer each traffic flow is uniquely identified by a Connection Identifier (CID). The bandwidth is requested to the BS on a CID-basis, while it is granted on an SS-basis. The BS has the task to schedule both uplink and downlink transmissions: the latter generate from the scheduling of local data queues at the BS, while per-SS uplink grants are provided in reply to the received per-flow Bandwidth Requests (BRs). Then, the SS is free of distributing the assigned aggregated resources to its currently active connections.

Five QoS classes are defined at the MAC layer [1] that provides a per-class differentiated traffic treatment. Unsolicited Grant Service (UGS) supports real-time constant bit rate applications. The BS assigns them unsolicited fixed bandwidth grants on the uplink at periodic intervals, based on the Maximum Sustained Traffic Rate. Real Time Polling Service (rtPS) is for real-time applications that produce variable size packets on a periodic basis. Extended Real Time Polling Services (ertPS) is similar in nature to rtPS service, but the BS grants to these flows a default uplink bandwidth and dynamically provides additional resources. Non Real Time Polling Services (nrtPS) is for delay-tolerant applications that generate variable size data packets and require a minimum data rate. Best Effort (BE) is appropriate to traffic with weak QoS requests. BE flows use contention slots to deliver their bandwidth requests.
TABLE I

WIMAX BURST PROFILES FOR WIRELESSMAN-OFDM PHY LAYER (CHANNEL BANDWIDTH 7MHz - BER $<10^{-6}$ )

\begin{tabular}{|c|c|c|c|c|}
\hline Modulation & $\begin{array}{c}\text { Overall } \\
\text { coding } \\
\text { rate }\end{array}$ & $\begin{array}{c}\text { Receiver } \\
\text { SNR } \\
{[\mathrm{dB}]}\end{array}$ & $\begin{array}{c}\text { Coded } \\
\text { block size } \\
\text { [byte] }\end{array}$ & $\begin{array}{c}\text { Receiver } \\
\text { Sensitivity } \\
{[\mathrm{dBm}]}\end{array}$ \\
\hline BPSK & $1 / 2$ & 6.4 & 24 & -88 \\
\hline \multirow{2}{*}{ QPSK } & $1 / 2$ & 9.4 & 48 & -85 \\
& $3 / 4$ & 11.2 & 48 & -83 \\
\hline \multirow{2}{*}{ 16QAM } & $1 / 2$ & 16.4 & 96 & -78 \\
& $3 / 4$ & 18.2 & 96 & -76 \\
\hline \multirow{2}{*}{ 64QAM } & $2 / 3$ & 22.7 & 144 & -71 \\
& $3 / 4$ & 24.2 & 144 & -70 \\
\hline
\end{tabular}

For each service class a set of mandatory QoS parameters are specified, among these we consider in our proposal:

- Minimum Reserved Traffic Rate (MRTR): the minimum data rate reserved for a service flow;

- Maximum Sustained Traffic Rate (MSTR): the peak data rate of the service flow.

Adaptive modulation and coding (AMC) techniques can be used in order to cope with the variability of radio links. The main task of AMC is to dynamically choose the modulation and coding scheme (MCS) that best meets the target Forward Error Correction (FEC) block error rate. Configurations of modulation/coding schemes in WiMAX are referred to as burst profiles [1], and are listed in Table I.

The BS determines the suitable MCS for each BS-to-SS transmission by comparing the monitored channel quality against the allowed values of Signal-to-Noise Ratio (SNR) and minimum input level sensitivity $\left(R_{S S}\right)$ for each burst profile at a given bit error rate (BER). $R_{S S}$ values are reported in Table I. They have been calculated as specified in [1], for a BER measured after the FEC block lower than $10^{-6}$ for standard message and test conditions, as in the following:

$$
R_{S S}=-102+S N R_{R X}+10 \cdot \log \left(F_{S} \cdot \frac{N_{\text {used }}}{N_{F F T}} \cdot \frac{N_{\text {subchannels }}}{16}\right)
$$

where:

- $S N R_{R X}$ is the SNR at receiver, reported in Table I;

- $F_{S}$ is the sampling frequency [MHz] calculated as $F_{S}=$ floor $(n \cdot B W / 8000) \cdot 8000$, where $B W$ is the nominal channel bandwidth and $n$ is a sampling factor equal to 8/7 with $7 \mathrm{MHz}$ channels, as suggested in [1];

- $N_{\text {used }}$ is the number of used subcarriers;

- $N_{F F T}$ is the smallest power of two greater than $N_{\text {used }}$;

- $N_{\text {subchannels }}$ is the number of allocated subchannels (default is 16 if no subchannelization is used).

When the received signal level for a given profile and target BER is below the receiver sensitivity, a more robust transport mode should be chosen, if available. A channel can be used for transmission as long as there is a burst profile which is capable to cope with its current status. Otherwise, if the signal level is below the receiver sensitivity even for the most robust burst profile (BPSK modulation with $1 / 2$ coding rate), then the radio link is too poor to be used for packet transmission. 


\section{The Proposed Radio Resource Management FRAMEWORK}

The proposed framework for providing QoS and fairness in WiMAX networks is based on a cross-layer design that relies on a strong interaction between MAC and PHY layers. The information about the radio link quality provided by the PHY layer is used by the MAC layer not only for selecting the subset of flows that can obtain service, but also for determining the resource assignment. The proposed RRM policy is implemented in the BS to efficiently manage the uplink bandwidth requests from the SSs. It is based on the following features:

- Channel status monitoring;

- Fair service provisioning to flows belonging to the same traffic class (intra-class fairness);

- Differentiated service provisioning to flows belonging to different traffic classes (inter-class QoS differentiation);

- Minimum reserved traffic rate guarantee to flows belonging to rtPS and nrtPS traffic classes;

- Possible delivery of the maximum sustained traffic rate to all flows;

- Optimal utilization of network resources.

Two RRM policies are presented, namely Minimum Assured Network Optimization (MANO) and Minimum Assured Weighted Allocation (MAWA). Both follows the same scheme:

1) Allocation of the minimum reserved rate and management of temporary peaks of requests. By peak of requests we refer to the situation in which the sum of the MRTR requests from the active rtPS and nrtPS flows is higher than the available bandwidth.

2) Compensation of the missed transmission opportunities. The BS keeps memory of the traffic flows that have been prevented to transmit due to poor channel conditions, and compensate for the missed opportunity as soon as the link quality is restored.

3) Allocation of the extra available bandwidth. The BS distributes among the requesting flows any residual bandwidth still available after the MRTR assignment and the compensation phase.

The difference between MANO and MAWA is in the way they take decisions at each of the above-mentioned steps. While MANO targets the overall network capacity by privileging requests from SSs with better channel conditions regardless of the traffic class, MAWA aims at favouring user satisfaction by assigning an amount of bandwidth proportional to the flow request regardless of the current link quality.

The scheduling decisions are taken on the basis of the information that the BS maintains in a data matrix. In particular, the matrix has a number of rows equal to the number of active service flows $N$ and keeps, for each flow, both static and time-varying information. Static information are set during the connection admission control phase and never modified during the network operation, while dynamic information are modified frame by frame on the basis of the channel status and the received bandwidth requests. The information stored in the data matrix are:

- the Class Of Service (CoS) for the flow (0 for BE, 1 for nrtPS, 2 for rtPS); UGS and ertPS flows are not considered since they receive a fixed amount of resources at each frame;

- the $M C S_{k}$ (with $k=0, \ldots, 6$ and $M C S_{0}=B P S K 1 / 2$ and $M C S_{6}=64 Q A M 3 / 4$ ) that the SS will use for uplink transmission based on the channel quality monitored by the BS;

- the Minimum Grant $(M G)$, in bytes, for meeting the minimum rate requirement for the flow;

- the Bandwidth Request (BR), in bytes, for the flow;

- the Normalized Minimum Grant (NMG) and Normalized Bandwidth Request (NBR) that represent, respectively, normalized values of $M G$ and $B R$, computed as explained in Step 0;

- the Compensation Counter (CC) that accounts for the number of past frames in which the traffic flow has not received the $M G$ because of adverse channel conditions.

Step 0. Preliminary operations: Minimum Grant computation

Before starting with Step 1 for both MANO and MAWA policies, the BS calculates (i) the per-flow $M G$ values, (ii) the available network capacity, and (iii) the normalized perflow $N M G$ and $N B R$ values.

The $M G$ values represent the minimum number of requested bytes per frame; they are computed from the MRTR requests of each rtPS and nrtPS flow as:

$$
\begin{array}{r}
M G_{i}=M R T R_{i} \cdot T_{\text {frame }} \\
i \in\{r t P S, n r t P S\}
\end{array}
$$

where $T_{\text {frame }}$ is the uplink subframe duration. The available capacity $C_{\text {available }}$ is calculated on the basis of the number of symbols $N_{\text {symb }}^{U L}$ that are allocated to the uplink direction (this parameter can be tuned by the BS according to the traffic load) and of the coded block size $(C B S)$ of the least robust MCS as in the following:

$$
C_{\text {available }}=N_{\text {symb }}^{U L} \cdot C B S_{64 Q A M 3 / 4}
$$

Since the same amount of bytes (requested or to be assigned) need to be transmitted with different data rates (on the basis of the perceived channel quality) then occupying a different amount of resources, we normalize $M G$ and $B R$ values using 64QAM modulation with $3 / 4$ coding rate as the reference burst profile, in order to have a fair comparison. The normalized per-flow $N M G$ and $N B R$ values are computed as:

$$
\begin{array}{r}
N M G_{i}=M G_{i} \cdot \frac{C B S_{64 Q A M 3 / 4}}{C B S_{i}} \\
i \in\{r t P S, n r t P S\} \\
N B R_{i}=B R_{i} \cdot \frac{C B S_{64 Q A M 3 / 4}}{C B S_{i}} \\
i \in\{r t P S, n r t P S, B E\}
\end{array}
$$


While the $N B R$ values are set for all active data flows, the $M G$ and $N M G$ variables are relevant only to flows requesting a minimum QoS assurance. It is also important to highlight that the $C_{\text {available }}$ value and the normalized variables $N M G$ and $N B R$ are defined by considering the 64QAM modulation with $3 / 4$ coding rate as the reference burst profile.

\section{Step 1. Allocation of Minimum Reserved Traffic Rate}

Both MANO and MAWA algorithms start by attempting to assign the $M G$ resources necessary to meet the negotiated MRTR to all rtPS and nrtPS active traffic flows. The resources are assigned in priority order, by starting from the rtPS class and then proceeding with nrtPS until network capacity is available. If there is sufficient bandwidth, at the end of this round, all rtPS and nrtPS active flows will have the requested minimum resources assigned for the current uplink frame.

Otherwise, if the requested bandwidth cannot be assigned because network resources are not sufficient or some radio link conditions are too poor, the two algorithms behave differently:

- In the case of MANO, the BS allocates resources starting from the flows with better channel conditions (regardless of the traffic class) and proceeds in decreasing order of channel state until network resources are available.

- In the case of MAWA, each flow $i$ is assigned a weight $W_{i}$ as the ratio between the normalized minimum grant needed for meeting the negotiated MRTR parameter and the total amount of minimum resources needed by all the flows belonging to the same class of flow $i$ equal to:

$$
\begin{array}{r}
W_{i}=\frac{\min \left(N M G_{i}, N B R_{i}\right)}{\sum_{j \in C_{i}, N B R_{j} !=0} \min \left(N M G_{j}, N B R_{j}\right)} \\
i \in\{r t P S, n r t P S, B E\}
\end{array}
$$

The BS distributes the available resources in traffic class priority order, from rtPS to nrtPS and to BE flows, proportionally to the per-flow weight $W_{i}$. Flows more disadvantaged by adverse past channel conditions will request more resources to compensate for missed transmission opportunities. Based on the weight used by the MAWA policy, these flows are able to obtain higher resource grants as soon as the channel quality recovers.

In summary, if MRTR cannot be guaranteed to all rtPS and nrtPS flows in a given uplink frame, in the MANO case only the subset of flows that gain service obtain the MRTR but some flows are not served at all, while in the MAWA case all the flows share the available bandwidth and get some resources, even if lower than the requested MG.

\section{Step 2. Compensation of missed transmissions}

This step has been designed with the aim of mitigating the effect that dissimilar wireless channel conditions can cause on flows that originate at different SSs. The compensation procedure is executed only if at the end of Step 1 some bandwidth is still available. Each time a rtPS or nrtPS flow $i$ cannot be provided with the requested $M R T R_{i}$ in Step 1 , its compensation counter $C C_{i}$ is incremented by one. When the channel condition for flow $i$ improves, during the execution of Step 2 it is assigned, if possible, a resource grant $C_{\text {comp }}^{i}$ equal to:

$$
\begin{array}{r}
C_{c o m p}^{i}=\min \left(C C_{i} \cdot N M G_{i}, N B R_{i}\right) \\
i \in\{r t P S, n r t P S\}
\end{array}
$$

If the available network capacity is not sufficient for compensating the missed transmission opportunities for all flows with non-negative $C C s$, resources are allocated in decreasing order of per-flow $C_{\text {comp }}$ values.

\section{Step 3. Allocation of extra bandwidth}

If further network resources are available after Step 2, they are allocated by following the same philosophy used in Step 1 for managing network resources when the MRTR cannot be provided to all rtPS and nrtPS flows. The difference is that while in Step 1 the BS operates on the total available network capacity, in Step 3 it distributes the residual network capacity.

\section{Performance AnAlysis}

Performance evaluation has been carried out by means of the $n s 2$ simulator, aiming at analyzing the effectiveness of the proposed channel-adaptive algorithms for QoS and fairness provisioning. Each simulation run has been repeated several times to get $95 \%$ confidence intervals in the presented results.

The reference topology is a $1000 \mathrm{~m} \times 1000 \mathrm{~m}$ grid with a WiMAX BS placed in the center of the grid and twelve SSs at different distances $d$ from the BS. For the sake of simplicity and without loss of generality, we assume that each SS generates only one flow of a given traffic class (rtPS, nrtPS, or BE), so the total number of active flows is twelve. Two scenarios are considered with different SS locations as specified in Table II. The locations are assigned to SSs in order to have, on average, the same channel conditions for all traffic classes. In Scenario 2 the average channel conditions are worse than in Scenario 1.

TABLE II

SCENARIOS SPECIFICATIONS

\begin{tabular}{|l|l|}
\hline \multicolumn{2}{|c|}{ Scenario 1 } \\
\hline $\mathbf{d}$ & Match between SS, CID and traffic class \\
\hline $100 \mathrm{~m}$ & SS1-1-BE, SS2-2-nrtPS, SS3-3-rtPS \\
\hline $200 \mathrm{~m}$ & SS4-4-BE, SS5-5-nrtPS, SS6-6-rtPS \\
\hline $300 \mathrm{~m}$ & SS7-7-BE, SS8-8-nrtPS, SS9-9-rtPS \\
\hline $400 \mathrm{~m}$ & SS10-10-BE, SS11-11-nrtPS, SS12-12-rtPS \\
\hline \multicolumn{2}{|c|}{ Scenario 2 } \\
\hline $\mathbf{d}$ & Match between SS, CID and traffic class \\
\hline $100 \mathrm{~m}$ & SS1-1-BE, SS2-2-nrtPS, SS3-3-rtPS \\
\hline $300 \mathrm{~m}$ & SS4-4-BE, SS5-5-nrtPS, SS6-6-rtPS \\
\hline $500 \mathrm{~m}$ & SS7-7-BE, SS8-8-nrtPS, SS9-9-rtPS \\
\hline $700 \mathrm{~m}$ & SS10-10-BE, SS11-11-nrtPS, SS12-12-rtPS \\
\hline
\end{tabular}

The radio link conditions have been simulated by the Stanford University Interim (SUI-6) channel model [14] in hilly terrain with moderate-to-heavy tree densities (Type A). The model accounts for both macroscopic channel effects, such as path loss $P L$ and shadowing according to Equation 
8 , and microscopic effects, such as multipath fading, which is modeled as a tapped delay line with 3 taps with non-uniform delays and maximum Doppler frequency (in Table III).

$$
P L=20 \log _{10}\left(\frac{4 \pi d_{0}}{\lambda}\right)+10 \gamma \log _{10}\left(\frac{d}{d_{0}}\right)+X_{f}+X_{h}+s
$$

where $d$ is the distance between SS and BS antennas [m]; $d_{0}=100 \mathrm{~m} ; s$ is a $\log$ normally distributed variable that accounts for the shadow fading. The path loss exponent is $\gamma=(a-$ $\left.b \cdot h_{b}+c / h_{b}\right)$, the constant values used for $a, b$ and $c$ depend on the terrain type, and $h_{b}$ is the BS antenna height above ground. Correction factors $X_{f}$ and $X_{h}$ respectively account of an operating frequency outside $2.5 \mathrm{GHz}$ and the SS antenna height above ground $\left(h_{r}\right)$.

TABLE III

CHANNEL MODEL AND SIMULATION ASSUMPTIONS

\begin{tabular}{|l|l|}
\hline Channel model & SUI-6 Terrain Type A \\
\hline BS (omn.) antenna height $\left(h_{t}\right)$ & $20 \mathrm{~m}$ \\
\hline SS antenna height $\left(h_{r}\right)$ & $5 \mathrm{~m}$ \\
\hline BS power & $40 \mathrm{dBm}$ \\
\hline Frequency band & $3.5 \mathrm{GHz}$ \\
\hline a, b, c constants & $4.6,0.0075\left[\mathrm{~m}^{-1}\right], 12.6[\mathrm{~m}]$ \\
\hline Correction factors & $X_{f}=6.0 \cdot \log _{10}(f / 200)$ \\
& $X_{h}=-10.8 \cdot \log _{10}\left(h_{r} / 200\right)$ \\
\hline Shadowing & Log-normal standard dev. $9 \mathrm{~dB}$ \\
\hline Multipath fading & Tapped-delay line with 3 taps \\
\hline Channel bandwidth & $7 \mathrm{MHz}$ \\
\hline Frequency band & $3.5 \mathrm{GHz}$ \\
\hline DL/UL ratio & $200 / 303$ \\
\hline Cyclic prefix time & $1 \mu \mathrm{s}$ \\
\hline Frequency band & $3.5 \mathrm{GHz}$ \\
\hline No. of subcarriers & 256 \\
\hline Frame duration & $10 \mathrm{~ms}$ \\
\hline Simulation duration & $5 \mathrm{~s}(500$ frames $)$ \\
\hline Duplexing mode & TDD \\
\hline Grid size & $1000 \mathrm{~m} \times 1000 \mathrm{~m}$ \\
\hline Number of nodes & $13(12 \mathrm{SSs}$ and $1 \mathrm{BS})$ \\
\hline
\end{tabular}

The uplink subframe is assigned a maximum number of 200 OFDM symbols out of the 303 symbols/frame available for each TDD frame [1] to account for the presence of downlink traffic. Under this assumption the maximum network throughput in the uplink direction goes from about $3 \mathrm{Mbps}$ (in the case of BPSK modulation with $1 / 2$ coding scheme) to more than 26Mbps (for 64QAM 3/4 MCS). Traffic type is Constant Bit Rate (CBR) and the simulated offered traffic load varies from $6 \mathrm{Mbps}(0.5 \mathrm{Mbps}$ for each of the twelve traffic flows) to $24 \mathrm{Mbps}$ (2Mbps per flow) to simulate a high congested network.

The analyzed performance parameters are:

- Network Capacity: is the number of data bytes carried by the WiMAX network during the simulation time;

- Goodput: is the per-flow achieved bit rate accounting for packets successfully delivered at the BS (i.e., without errors and within the maximum tolerated latency);

- Jain's Fairness Index (JFI): measures the intra-class fairness; $x_{i}$ is the goodput achieved by flow $i$ and $N$ is the number of competing flows in the same class. JFI is equal to 1 in the case of perfect fairness [15].

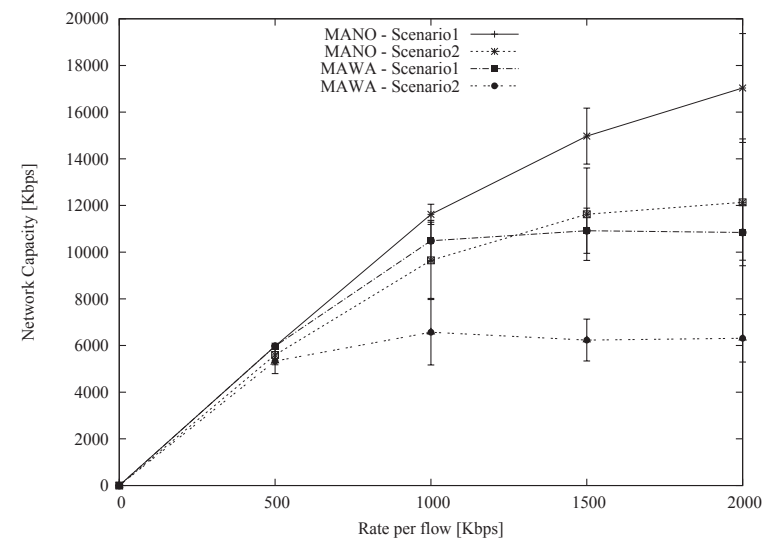

Fig. 1. Network Capacity of MANO and MAWA in Scenario 1 and Scenario 2 under variable traffic load.

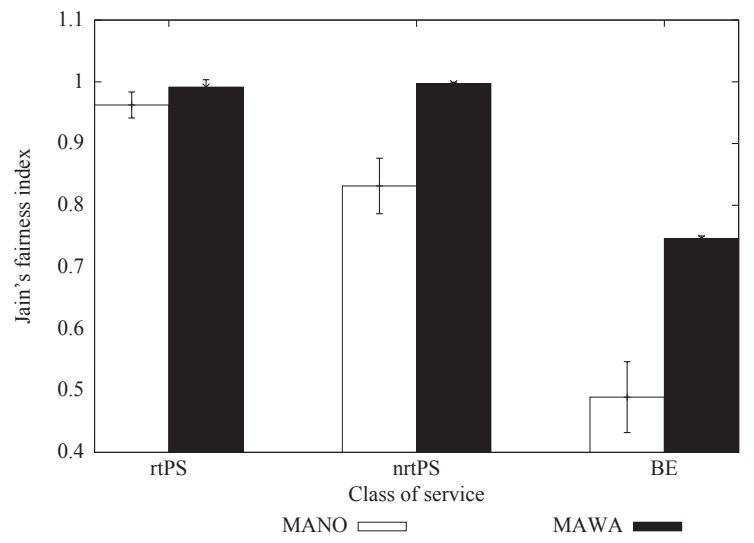

Fig. 2. JFI of $M A N O$ and $M A W A$ in Scenario 1 for a network load equal to $24 \mathrm{Mbps}$.

$$
J F I=\frac{\left(\sum_{i=1}^{N} x_{i}\right)^{2}}{N \sum_{i=1}^{N}\left(x_{i}\right)^{2}}
$$

Figure 1 shows the network capacity achieved by MANO and MAWA in Scenarios 1 and 2 when the offered traffic load goes from $6 \mathrm{Mbps}$ to $24 \mathrm{Mbps}$. In both scenarios MANO significantly outperforms MAWA due to the fact that MANO privileges flows with best channels and then higher data rate. The performance of both algorithms is strongly dependent on the channel conditions; in Scenario 2 in fact performances are worse than in Scenario 1.

The higher network capacity provided by MANO is at the expense of a lower JFI for all the scheduled traffic classes, as shown in Figure 2. This is again due to the different philosophies of the two approaches. MAWA aims at achieving proportional bandwidth assignment that accounts for missed transmission opportunities by assigning a higher weight to flows that have suffered more in the past frames.

The goodput provided by MANO and MAWA to each single rtPS and nrtPS traffic flow is shown, respectively, in Figure 
3 (a) and (b) when considering Scenario 1 and a traffic load equal to $24 \mathrm{Mbps}$. Both MANO and MAWA can differentiate the treatment of rtPS and nrtPS flows by guaranteeing to real time traffic higher goodput than to nrtPS traffic. Furthermore, the goodput guaranteed by MANO depends on the per-flow channel state, so flows originated by SSs farther from the BS achieve lower performances than flows originated by SS closer to the BS. On the contrary, MAWA is able to guarantee intraclass fairness in terms of achieved goodput, but the value of goodput is lower than in the case of MANO.

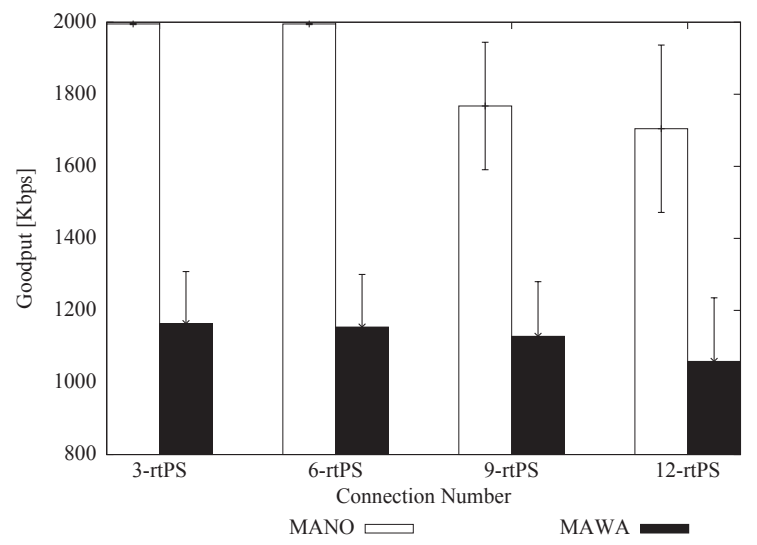

(a)

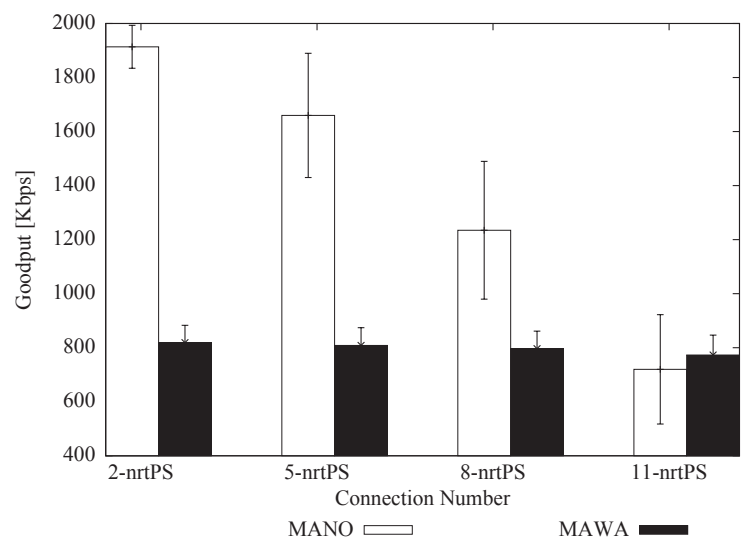

(b)

Fig. 3. Goodput of (a) rtPS and (b) nrtPS flows for MANO and MAWA in Scenario 1 for a network load equal to $24 \mathrm{Mbps}$.

Finally, the benefits of the compensation mechanism implemented in Step 2 are shown through the analysis of the goodput achieved by rtPS flows in Table IV. It is worth noting that the flow affected by the most adverse channel conditions (i.e., flow 12 ) is the one gaining more advantage from the compensation mechanism. This is at the expense of the other flows that undergo a very slight performance decrease. This fairness improvement is paid in terms of a small network capacity decrease, due to the necessity to serve a flow that, likely, will be transmitted by using a robust MCS, thus utilizing a high quantity of network resources.
TABLE IV

GOODPUT GAIN OF MANO DUE TO COMPENSATION (RTPS FLOWS IN SCENARIO 1).

\begin{tabular}{|c|c|c|}
\hline Flow ID & Distance [m] & Goodput gain [\%] \\
\hline Flow 3 & 100 & -0.046 \\
\hline Flow 6 & 200 & -0.046 \\
\hline Flow 9 & 300 & -0.110 \\
\hline Flow 12 & 400 & +2.610 \\
\hline
\end{tabular}

\section{CONCLUSIONS}

In this paper we designed a channel-adaptive QoS-support framework to be implemented in the WiMAX Base Station for assignment of uplink radio resources to fixed subscriber stations. The BS generates bandwidth grants on the basis of information on channel quality, service requirements, and past treatment of the traffic flow, with the aim of providing intra-class fairness and QoS differentiation with a minimum performance assurance. A compensation technique has been designed that aims at mitigating the negative effects caused by fluctuations in the wireless channel.

Achieved simulation results show the effectiveness of the proposed framework and highlight a clear tradeoff between network capacity and flow fairness for the two proposed approaches.

\section{REFERENCES}

[1] IEEE 802.16 - Standard for local and metropolitan area networks, Part 16: Air Interface for Broadband Wireless Access Systems, 2009.

[2] M. A. Teixeira, and P.R. Guardieiro, "A predictive scheduling algorithm for the uplink traffic in IEEE 802.16 networks", ICACT 2010.

[3] Y. P. Fallah, P. Nasiopoulos, and R. Sengupta, "Fair scheduling for realtime multimedia support in IEEE 802.16 wireless access networks", WoWMoM 2010.

[4] Z. Yurdakul, and S. Oktug, "A Hierarchical Channel-Aware Uplink Scheduler for WiMAX Base Stations", AICT 2010.

[5] A. Iera, A. Molinaro, and S. Pizzi, "Channel-Aware Scheduling for QoS and Fairness Provisioning in IEEE 802.16/WiMAX Broadband Wireless Access Systems", IEEE Network, 2007, 21:5, pp. 34-41.

[6] F.A. Kubota, J.F. Borin, and N.L.S. da Fonseca, "Opportunistic Crosslayer Uplink Scheduler for the IEEE 802.16 standard", ICC 2011.

[7] C. So-In, R. Jain, and A.-K. Tamimi, "Scheduling in IEEE 802.16e mobile WiMAX networks: key issues and a survey", IEEE Journal on Selected Areas in Communications, 2009, 27:2, pp. 156-171.

[8] N. Abu Ali, P. Dhrona, and H. Hassanein, "A performance study of uplink scheduling algorithms in point-to-multipoint WiMAX networks", Computer Communications, 2009, 32:3, pp. 511-521.

[9] C. Cicconetti, A. Erta, L. Lenzini, and E. Mingozzi, "Performance evaluation of the IEEE 802.16 MAC for QoS support", IEEE Transactions on Mobile Computing, 2007, 6:1, pp. 2638.

[10] P. Rengaraju, C.-H. Lung, and A. Srinivasan, "QoS Assured Uplink Scheduler for WiMAX Networks", VTC 2010-Fall.

[11] W. Nie, H. Wang, and N. Xiong, "Low-overhead uplink scheduling through load prediction for WiMAX real-time services", IET Commununications, 2011, 5:8, pp. 1060-1067.

[12] R. Garroppo, S. Giordano, D. Iacono, and L. Tavanti, "Game Theory and time utility functions for a radio aware scheduling algorithm for WiMAX networks", Wireless Networks, 2011, 17:6, pp. 1441-1459.

[13] Y. Chiu, C. Chang, K. Feng, and F. Ren, "GGRA: A Feasible ResourceAllocation Scheme by Optimization Technique for IEEE 802.16 Uplink Systems", IEEE Transactions on Vehicular Technology, 2010, 59:3, pp. 1393-1401.

[14] "Channel models for fixed wireless applications", IEEE 802.16 Broadband Wireless Access Working Group, June 2003.

[15] R. Jain, D. Chiu, and W. Hawe, "A Quantitative Measure of Fairness and Discrimination for Resource Allocation in Shared Computer Systems”, DEC Research Report TR-301, September 1984. 